\author{
Erwin J. O. Kompanje \\ Nichon E. Jansen
}

\section{Reglaze your glasses!}

\author{
The unused potential of organ donors \\ in times of high demand
}

Received: 23 June 2014

Accepted: 4 July 2014

Published online: 5 August 2014

(C) Springer-Verlag Berlin Heidelberg and ESICM 2014

\section{E. J. O. Kompanje (ه)}

Department of Intensive Care Medicine, Erasmus MC University Medical Center Rotterdam, P.O. Box 2040, 3000 CA Rotterdam, The Netherlands

e-mail: e.j.o.kompanje@erasmusmc.nl

Tel.: +31-6-53837655

\section{N. E. Jansen}

Dutch Transplant Foundation, Leiden, The Netherlands

One of the constant struggles of transplantation medicine is to obtain enough organs to transplant. The demand for organ transplantation has continued to rise. The three main sources of donor organs are (1) the brain-dead multiorgan donor, (2) the circulatory arrest donor and (3) the living donor.

Brain-dead patients are the ideal donors and the only source for hearts. Ideally, as warm ischaemia does not influence the time in which organs must be harvested after declaration of death. However, brain death is an undesirable outcome of intensive care medicine, resulting from the ability to prolong and distort the process of dying in patients with acute and severe cerebral damage. The availability of brain-dead donors is highly susceptible to changes and differences in its causes and on country and time period. In many Western countries, the brain-dead patient has become a rare outcome of intensive care. The actual numbers are declining. The most important reason for this is the decline in incidence and mortality of subarachnoid haemorrhage and traumatic brain injury. The circulatory death donor is a donor who does not fulfil brain death criteria, but is eligible for organ donation after circulatory arrest. Practical challenges include identification of patients who will die within $2 \mathrm{~h}$ after withdrawal of life-sustaining measures, support and maintaining the trust of relatives of the dying patient and society [1,2], and ethical and legal acceptance of the practice in the different countries of the world. Although many patients
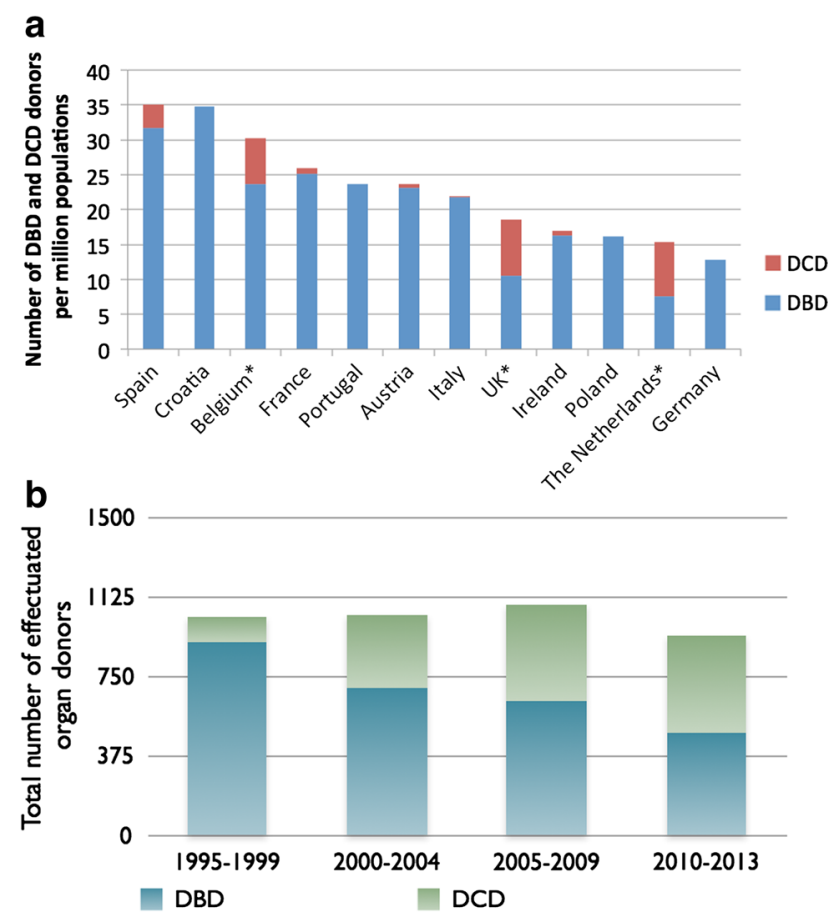

Fig. 1 a Distribution of donation after brain death (DBD) and donation after circulatory death (DCD) in European countries per million populations, 2012. Asterisk indicates mostly controlled DCD. b Comparison of effectuated DCD and DBD in different time eras in the Netherlands over the past 18 years (database Dutch Transplant Foundation) 
who die after controlled withdrawal of life-sustaining measures and donate their organs suffered from neurological conditions, patients with a primary diagnosis of respiratory disease are also effectuated as organ donors.

In some countries (e.g. the Netherlands and UK) a substantial number of donors have died after controlled circulatory arrest [1]. In others, like Spain, this is only a small percentage of all deceased organ donors (Fig. 1). But, intensive care mortality of patients with subarachnoid haemorrhage is higher than in other countries and more of these patients progress to brain death [3]. In the Netherlands, the number of effectuated donations after declaration of brain death has declined from 915 in the period 1995-1999 to 486 patients in the period 2010-2013. The number of circulatory death donors increased from 118 patients in the first time period to 458 in the last period. Between 1995 and 1999, 88.6\% of the total number of deceased organ donors were brain-dead donors; between 2010 and 2013 this declined to $51.4 \%$. Between 1995 and 1999, only $11.4 \%$ of all deceased organs donors donated after circulatory death. Between 2010 and 2013 this has increased to $48.5 \%$ (Fig. 1). Most probably, in the coming years, there will be more deceased organ donors in the Netherlands who are declared dead after circulatory death than after brain death. In the UK, the number of DCD donors increased from 199 to 373 between 2007 and 2012, an increase of $87 \%$ [4].

DCD provides a valuable source of donor organs and is a solution for the shortfall between supply and demand. Most donations after circulatory death take place after withdrawal of life-sustaining measures in non-brain-dead patients in the intensive care unit (ICU). It offers the possibility to facilitate the wishes of patient and/or relatives at the end of life in patients who are not brain dead. The majority of organ donations after circulatory death are controlled procedures after planned withdrawal of mechanical ventilation in consultation with the relatives of the patient. Although the quality of donor organs from circulatory death donors is highly improved, progress can still be made. For example, implementation of a DCD protocol using extracorporeal perfusion can lead to a further increase in donor organs of high quality [5]. As the use of Maastricht category 3 DCD donors is associated with withdrawal of life-sustaining measures in the ICU, differences between countries and hospitals are inescapable. In countries and hospitals with a more liberal policy of withdrawal of life-sustaining measures, a DCD protocol can be more easily implemented than in countries in which end-of-life decision-making is more debatable. Another barrier for the use of DCD donors after withdrawal of life-sustaining measures is the short time window between circulatory arrest and transport to the operating theatre for harvesting the organs. This short 'hands-off' time window, usually $5 \mathrm{~min}$, can be burdensome for the relatives, who want to spend some time beside their deceased loved one.

As the incidence and mortality of subarachnoid haemorrhage is declining in most Western countries [6, 7], it is plausible to assume that the number of brain-dead organ donors will further decrease. The decline in incidence and mortality of conditions leading to the state of brain death (the leading causes being subarachnoid haemorrhage and traumatic brain injury) is desirable, but also inescapable [8]. This decline is a good thing seen from interests of patients, their relatives and society, but has serious consequences for patients waiting for donor organ transplantation. All countries that are faced with declining numbers of brain-dead organ donors should expand their horizons. In many European countries the use of organ donors after circulatory death in a controlled situation is non-existent (e.g. Germany, France, Italy, Finland, Lithuania, Bosnia-Herzegovina, Greece, Hungary, Norway, Ireland, Austria, Portugal and Switzerland) [9].

In this issue of Intensive Care Medicine, Lesieur et al. [10] describe the eligibility for organ donation following withdrawal of life-sustaining measures in 43 French ICUs. Although uncontrolled DCD is done in France on a small scale (in 2012, 53 donors, equivalent to 0.8 per million population), organ donation after controlled circulatory death is still not legally framed in France and, although it was debated in the French parliament in 2013, it is still not practised. They found a significant number of patients in French ICUs that would have been eligible for organ donation after circulatory death. Some 5,000 organ transplants were performed in France in 2012. However, more than 16,000 patients were on the waiting list. Every increase in the total number of donor organs and every decline of number of patients on the waiting list is highly desirable. The study by Lesieur et al. proved that this potential is available but still not used. Their results can be used to convince the French parliament to "reglaze their glasses" when looking at the sources of organ donation and that allowing this form of organ donation will save the lives and/or improve the quality of life of many French civilians, not only in France. In other European countries the decline of brain-dead organ donors is progressive and inescapable, and the need for other sources of donor organs is striking. The initiative of Lesieur et al. [10] should be followed by other scholars in European countries in which donation after controlled circulatory death is still not being done or where potential donors are missed [11], but where, as in many other countries, there is a widening gap between supply and demand of donor organs. 


\section{References}

1. Council of Europe (2013) International figures on organ donation and transplantation 2012. Newsletter Transplant 18:34-36

2. Shemie SD, Hornby L, Baker A, Teitelbaum J, Torrance S, Young K, Capron AM, Bernat JL, Noel L (2014) International guideline development for the determination of death. Intensive Care Med 40(6):788-797

3. Mourelo-Fariña M, Aller-Fernández A, Vidal-Cortés P, Galeiras R, Gracía M (2011) Spontaneous subarachnoid hemorrhage: clinical impact, prognostic value and complications. Crit Care 15(Suppl 1):P326

4. NHS Blood and Transplant (2014) Transplant activity in the UK, activity report. Available via https:// www.organdonation.nhs.uk/ukt/.../ transplant_activity_report/. Accessed 1 Jan 2014
5. Barrau B, Billault C, Nicolas-Robin A (2013) The use of extracorporeal membranous oxygenation in donors after cardiac arrest. Curr Opin Organ Transplant 18(2):148-153

6. Stegmayr B, Eriksson M, Asplund K (2004) Declining mortality from subarachnoid haemorrhage: changes in incidence and case fatality from 1985 through 2000. Stroke 35:2059-2063

7. Locelock CE, Rinkel GJ, Rothwell PM (2010) Time trends in outcome of subarachnoid haemorrhage. Neurology 74:1494-1501

8. Kompanje EJO, De Groot YJ, Bakker J (2011) Is organ donation from brain dead donors reaching an inescapable and desirable nadir? Transplantation 91:1177-1180
9. Dominguez-Gil B, Haase-Kromwijk BJJM, Van Leiden HA et al (2011) Current situation of donation after circulatory death in European countries. Transpl Int 24:676-686

10. Lesieur O, Leloup M, Gonzalez F et al (2014) Eligibility for organ donation following end-of-life decisions: a study performed in 43 French Intensive Care Units. Intensive Care Med. doi: 10.1007/s00134-014-3409-2

11. Kutsogiannis DJ, Asthana S, Townsend DR, Singh G, Karvellas CJ (2013) The incidence of potential missed organ donors in intensive care units and emergency rooms: a retrospective cohort. Intensive Care Med 39(8):1452-1459 